NIST Technical Note 2057

\title{
Effect of Strain Rate on Glass Fiber Failure Process in a Glass Fiber/Epoxy Model Composite
}

Jae Hyun Kim

Steven P. Mates

N. Alan Heckert Jeremiah W. Woodcock

Gale A. Holmes

This publication is available free of charge from:

https://doi.org/10.6028/NIST.TN.2057

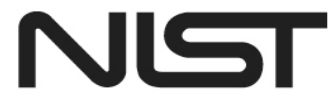

National Institute of Standards and Technology U.S. Department of Commerce 


\title{
Effect of Strain Rate on Glass Fiber Failure Process in a Glass Fiber/Epoxy Model Composite
}

\author{
Jae Hyun Kim \\ Materials and Structural Systems Division \\ Engineering Laboratory \\ Steven P. Mates \\ Jeremiah W. Woodcock \\ Gale A. Holmes \\ Materials Science and Engineering Division \\ Materials Measurement Laboratory \\ N. Alan Heckert \\ Statistical Engineering Division \\ Information Technology Laboratory
}

This publication is available free of charge from:

https://doi.org/10.6028/NIST.TN.2057

September 2019

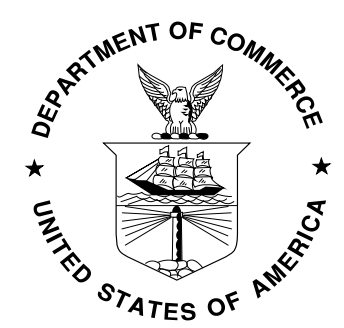

U.S. Department of Commerce Wilbur L. Ross, Jr., Secretary

National Institute of Standards and Technology Walter Copan, NIST Director and Undersecretary of Commerce for Standards and Technology 
Certain commercial entities, equipment, or materials may be identified in this document in order to describe an experimental procedure or concept adequately. Such identification is not intended to imply recommendation or endorsement by the National Institute of Standards and Technology, nor is it intended to imply that the entities, materials, or equipment are necessarily the best available for the purpose.

National Institute of Standards and Technology Technical Note 2057 Natl. Inst. Stand. Technol. Tech. Note 2057, 18 pages (September 2019) CODEN: NTNOEF

This publication is available free of charge from: https://doi.org/10.6028/NIST.TN.2057 


\begin{abstract}
In this study, we elongated glass-fiber model composites in tension to monitor local fiber breakage in the epoxy matrix under quasi-static loading (approximately $0.00001 \mathrm{~s}^{-1}$ ) and high rate loading (approximately $200 \mathrm{~s}^{-1}$ ). Strains for the initial fiber breaks were around $2 \%$ under the quasi-static loading, while the strains for the initial fiber breaks were around $4 \%$ to $5 \%$ for the high strain rate loading, indicating significant rate dependent characteristics on tensile failure strains of the glass fibers.
\end{abstract}

Key words : Glass fiber, epoxy, high strain rate, fragmentation test, Kolsky bar 


\section{Table of Contents}

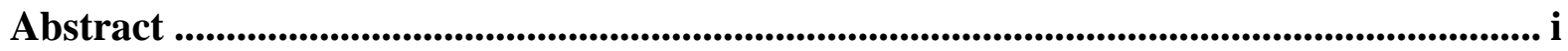

List of Tables .................................................................................................................. ii

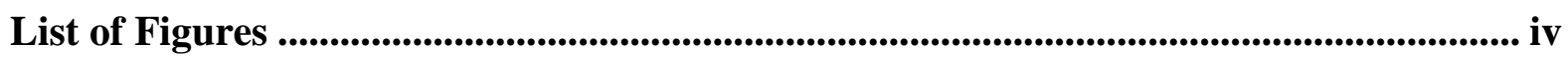

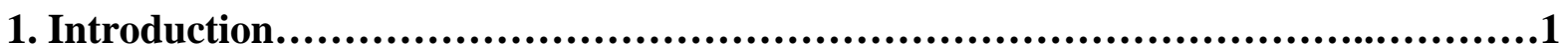

2. Experimental Materials and Test Methods........................................ 2

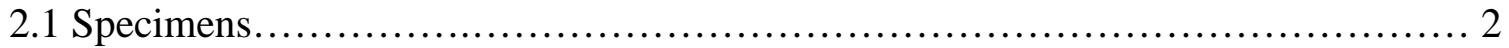

2.2 Mechanical and spectroscopic measurements ................................. 2

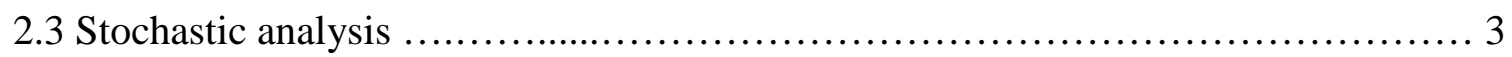

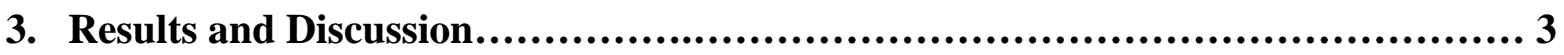

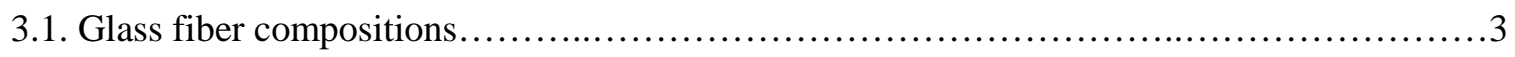

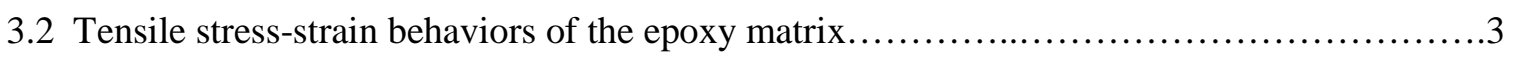

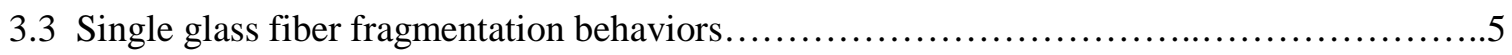

4. Conclusions and Future Work........................................................................6

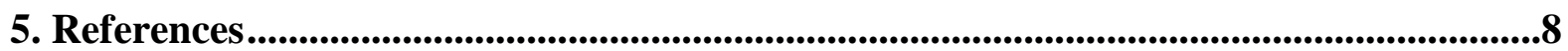

Appendix A: Supplemental Materials ...........................................................................................9 


\section{List of Tables}

Table 1. Atomic concentrations for the glass fiber after $24 \mathrm{~h}$ of piranha etching...............3 


\section{List of Figures}

Figure 1. Fiber fragmentation tests using a manual tensile apparatus (a) and Kolsky tension

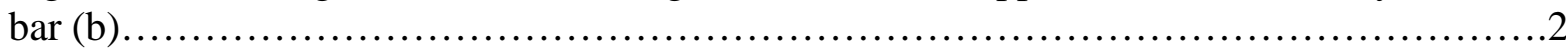

Figure 2. Typical stress-strain behaviors of the single fiber composites for the $17 \%$ DGEBD - epoxy (a) and 34 \% DGEBD - epoxy (b) for the quasi-static (dotted lines with symbols) and HSR (solid lines) during the fragmentation tests with DIC images (c) of the HSR tests. Stress-strain curves up to reaching saturations are exhibited and individual symbols indicate replicates. Error bars on HSR tests are $\pm 4 \%$ on engineering stress and \pm 2 standard deviations of the averaged 3DDIC measured engineering strains over the gauge section.....4

Figure 3. Evolution of the number of fiber breaks with engineering strains using the $17 \%$ DGEBD-epoxy (a) and 34 \% DGEBD-epoxy (b) matrix for the quasi-static (filled) and the HSR (hollow) loading conditions with the microscopic images of the fiber breaks in the $34 \%$ DGEBD-epoxy matrix(c)...................................................

Figure 4. Probability Plot Correlation Coefficient (PPCC) comparison for the uniform (blue) and 3-parameter Weibull (red) distributions of the $17 \%$ DGEBD-epoxy (a, c) and $34 \%$ DGEBD-epoxy (b, d) specimens under the quasi-static (a, b) and HSR (c, d) are shown with the lines for the average PPCC obtained by the entire tests. Fragmentation tests with fewer than five breaks are omitted for the PPCC comparison due to insufficient sample size........6 
List of Acronyms and Abbreviations

HSR

DGEBA

DGEBD

m-PDA

DIC

XPS

PPCC
High strain rate

diglycidyl ether of bisphenol-A 1,4-butanediol diglycidyl ether meta-phenylene diamine digital image correlation x-ray photoelectron spectroscopy probability plot correlation coefficient 


\section{Introduction}

Glass fiber reinforced composites with high strength and light weight are widely used in various civil engineering and automotive applications [1, 2]. When the glass fiber composites are subjected to various rates for loading conditions (from $\approx 0.001 \mathrm{~s}^{-1}$ of quasistatic to $\approx 100 \mathrm{~s}^{-1}$ of high strain rate (HSR)) in such applications, the sensitivities of the strain rate on the mechanical behavior of glass fiber composites are important parameters to design structures that would not fail unexpectedly at HSR loading. Various test methods using drop towers, Hopkinson bars, and hydraulic machines [3] have been applied to conduct HSR tests, but HSR data on the tensile mechanical properties of the glass fiber composites are relatively limited compared to those for the quasi-static test data. According to the literatures, the tensile strengths for unidirectional glass fiber epoxy composites were significantly increased by increasing the strain rates from $0.001 \mathrm{~s}^{-1}$ to $100 \mathrm{~s}^{-1}$ while the tensile modulus and strain to failure were slightly increased [4]. In contrast, tensile modulus and failure stress for the angle ply glass / epoxy laminate were strain rate independent [5]. For the plain woven fabric geometry, the tensile strengths of the composites in the warp and fill directions measured around $400 \mathrm{~s}^{-1}$ were significantly increased compared with the quasi-static loading while the increase in the ultimate tensile strains were marginal [6], which implies a significant increase in moduli. Furthermore, finite element simulation of the glass fiber composite in fiber length direction showed no strain rate dependence in tension [7]. The ambiguity of the strain rate effects on the tensile mechanical properties of the glass fiber composites found in the literature review can be attributed to lack of systematic micro and macro level test data for damage initiation and propagation with various fiber array during HSR loading.

Compared to relatively well-defined tensile specimen geometries to observe a failure process at macro scales[8], tensile tests at micro scales typically use model composite systems with a single fiber totally encapsulated in a matrix coupon to visually observe a fiber failure process [9]. In tensile loading for the model composites, the interfacial shear stress transfer mechanism occurs as external tensile forces are transferred to the encapsulated fiber through the interface. When the fiber tensile strength is exceeded, the fiber fractures inside the matrix and this process is repeated producing shorter fragments with higher tensile loading until the fragment lengths are too short to produce additional fracture through the stress transfer mechanism. Typically, fiber strength and interfacial adhesion strength can be estimated from the test [10], and the model composite configuration with a single fiber was applied to study micromechanics of coordinated fiber failure in a multi-fiber array that is similar to the fiber array of the unidirectional fiber composites [11].

In this study, a model composite configuration that is frequently used for fiber/matrix interfacial stress characterizations was employed to study the effects of HSR loadings on the glass fiber failure process in epoxy matrix during tensile loading, which referred as a fragmentation test hereafter. A Kolsky bar test apparatus was used to apply HSR loading for the fragmentation tests while a manual loading device was used to apply the quasi-static loading. For both tests, the glass fiber failure locations in the epoxy matrix were measured using a microscope. After the fragmentation tests, stochastic analyses were performed for fiber break locations obtained by the HSR and quasi-static loading tests to investigate the loading rate effects on fiber failure process. 


\section{Experimental materials and test methods}

\subsection{Specimens}

Water sized glass fibers of $15 \mu \mathrm{m}$ to $16 \mu \mathrm{m}$ diameter were used and two single glass fibers were carefully placed in the middle of a silicon rubber mold at least $300 \mu \mathrm{m}$ apart under approximately $4.2 \mathrm{mN}$ of pretension. All glass fibers used in tensile tests were encapsulated in the epoxy without any additional treatment. Three different compositions of epoxy matrix systems were used including diglycidyl ether of bisphenol-A (DGEBA, MillerStephenson, ${ }^{1 * *}$ ), 1,4-butanediol diglycidyl ether (60 \% purity DGEBD, Sigma-Aldrich), and meta-phenylene diamine (m-PDA, Sigma-Aldrich). The epoxy matrix (i.e. controlled matrix) without DGEBD was prepared by mixing with $100 \mathrm{~g}$ of DGEBA and $17.5 \mathrm{~g}$ of mPDA. Two modified epoxy matrix systems (i.e., the $17 \%$ DGEBD - epoxy, and the $34 \%$ DGEBD epoxy) were prepared by mixing with 17 mass \% and 34 mass \% of DGEBD respectively. All model composites were cured at $60{ }^{\circ} \mathrm{C}$ for $3 \mathrm{~h}$. followed by $121{ }^{\circ} \mathrm{C}$ for $2 \mathrm{~h}$. to fabricate specimens with two holes for the pin grips (Figure 1a), and then elongated to perform the fiber fragmentation test [9]. The gauge length of the specimen used in the this study was 4 mm to achieve force equilibrium [12] and to achieve a peak strain of $5 \%$ to $6 \%$ in the tests.

\subsection{Mechanical and spectroscopic measurements}

The strain rates $(\dot{\varepsilon})$ of both quasi-static and HSR experiments were calculated using the following equations: $\dot{\varepsilon}=v / L$, where $v$ is the slope (velocity) of the displacement-time curves in a linear region and $L$ is the gauge length. For quasi-static loading experiments $(\approx$ $0.00001 \mathrm{~s}^{-1}$ ), the specimens were fixed using the metal pins on the grips and elongated approximately $0.025 \mathrm{~mm}$ per loading step (10 min. between the loading steps ) using a manual loading apparatus as shown in Figure 1a. Two fiducial marks (approximately $4 \mathrm{~mm}$ apart) on the specimen was used for strain measurements, and glass fiber fragmentation behaviors were observed until reaching saturation (i.e. no additional fragment formation) using a microscope with $20 \mathrm{x}$ objective lens. For the HSR loading experiments $\left(\approx 200 \mathrm{~s}^{-1}\right)$, a direct tension Kolsky bar was used (Figure 1b), a tension Kolsky bar was used (Figure 1b) with three-dimensional high speed digital image correlation (3DDIC) system. Fiber break locations were measured under microscope after completing HSR loading. The details are in supplements.

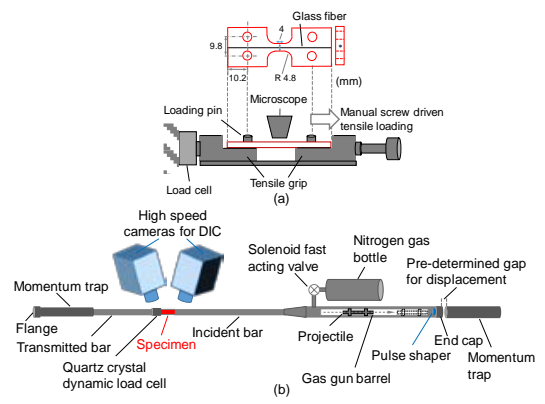

Figure 1. Fiber fragmentation tests using a manual tensile apparatus (a) and Kolsky tension bar (b)

\footnotetext{
** Certain commercial equipment, instruments, or materials are identified in this paper in order to specify the experimental procedure adequately. Such identification is not intended to imply recommendation or endorsement by the National Institute of Standards and Technology, nor is it intended to imply that the materials or equipment identified are necessarily the best available for the purpose
} 
To investigate the atomic composition of the glass fiber that was etched by piranha solution, x-ray photoelectron spectroscopy (XPS) was performed on a Kratos AXIS Ultra DLD spectrometer with a monochromated $\mathrm{Al} \mathrm{K}_{\alpha}$ source operating at $1486.6 \mathrm{eV}$ and $140 \mathrm{~W}$. the etched glass fibers were used only in the XPS measurements for the purpose of identifying the composition of the glass fibers.

\subsection{Stochastic analysis}

The stochastic analyses for the break locations obtained after completing the fragmentation tests (i.e., stress free state) were conducted using the 3-parameter Weibull and uniform distributions, and the probability plot correlation coefficient (PPCC) was used to assess the goodness-of-fit for the break locations. The details of the PPCC assessment can be found in the Ref [13].

\section{Results and Discussion}

\subsection{Glass fiber compositions}

XPS measurements shown in Table 1 report the composition in atomic percent on glass fibers surface etched in typical piranha solution (a mixture of sulfuric acid and hydrogen peroxide). The atomic compositions of the glass fiber used in this study doesn't include boron, which agrees well with those of the unsized E-glass fiber [14]. The presence of 16.6 at. \% of $\mathrm{C} 1 \mathrm{~s}$ is attributed to advantageous carbon in the form of pump oil in the instrument vacuum chamber or from sample handling, or, to residual carbon contamination of the glass fiber production process that is associated with $67 \% \mathrm{C}-\mathrm{C} / \mathrm{H}, 20 \% \mathrm{C}-\mathrm{O}-\mathrm{C} / \mathrm{H}, 5 \%$ $\mathrm{C}=\mathrm{O} / \mathrm{O}-\mathrm{C}-\mathrm{O}[14]$.

Table 1 Atomic concentrations for the glass fiber after $24 \mathrm{~h}$ of piranha etching.

\begin{tabular}{lccccccc}
\hline Nuclei & O 1s & C 1s & Ca 2p & Na 1s & Si 2p & Al 2p & Mg KLL \\
\hline Mean. Atomic \% & 51.5 & 16.6 & 4.1 & 0.7 & 16.0 & 5.0 & 6.0 \\
\hline
\end{tabular}

Note that the piranha etched glass fiber was used only for the XPS measurement.

\subsection{Tensile stress-strain behaviors of the epoxy matrix}

Figure 2a and $\mathrm{b}$ shows the stress-strain behaviors of model composites during fragmentation tests at both quasi-static and HSR loadings in tension. The controlled epoxy specimens without DGEBD generally failed at a stress level of about $110 \mathrm{MPa}$ before reaching $5 \%$ to $6 \%$ strain during the HSR tests, so the analysis of fiber damage was not performed in this epoxy matrix system. Both the $17 \%$ DGEBD-epoxy and 34 \% DGEBDepoxy matrices didn't fail at those strain levels and exhibited significant rate sensitivity particularly in the region $2 \%$ strain. For instance, the stress levels for the HSR tests at peak strain reach approximately $120 \mathrm{MPa}$, while the quasi-static tests are below $80 \mathrm{MPa}$ for similar strains, representing a $50 \%$ increase in strength at HSR, as shown in Figure 2. As indicated in the 3DDIC images for the HSR test (Figure 2c), the tensile strain reaches its peak value at approximately $150 \mu$ s after deformation begins (see the details in supplement). Since the tensile loadings for both quasi-static and HSR tests were stopped to observe the 
glass fiber break locations in the epoxy matrix, failure strains of the epoxy matrix were not determined here. The strain distribution is slightly non-uniform in the center of the specimen, due to the use of a short gauge length. Although a specimen design with a longer gauge length provides a wider range data for fiber failure locations, a Kolsky bar test needs to be designed in approximating a striker bar length and velocity to reach a desired strain for the mechanical tests [12].
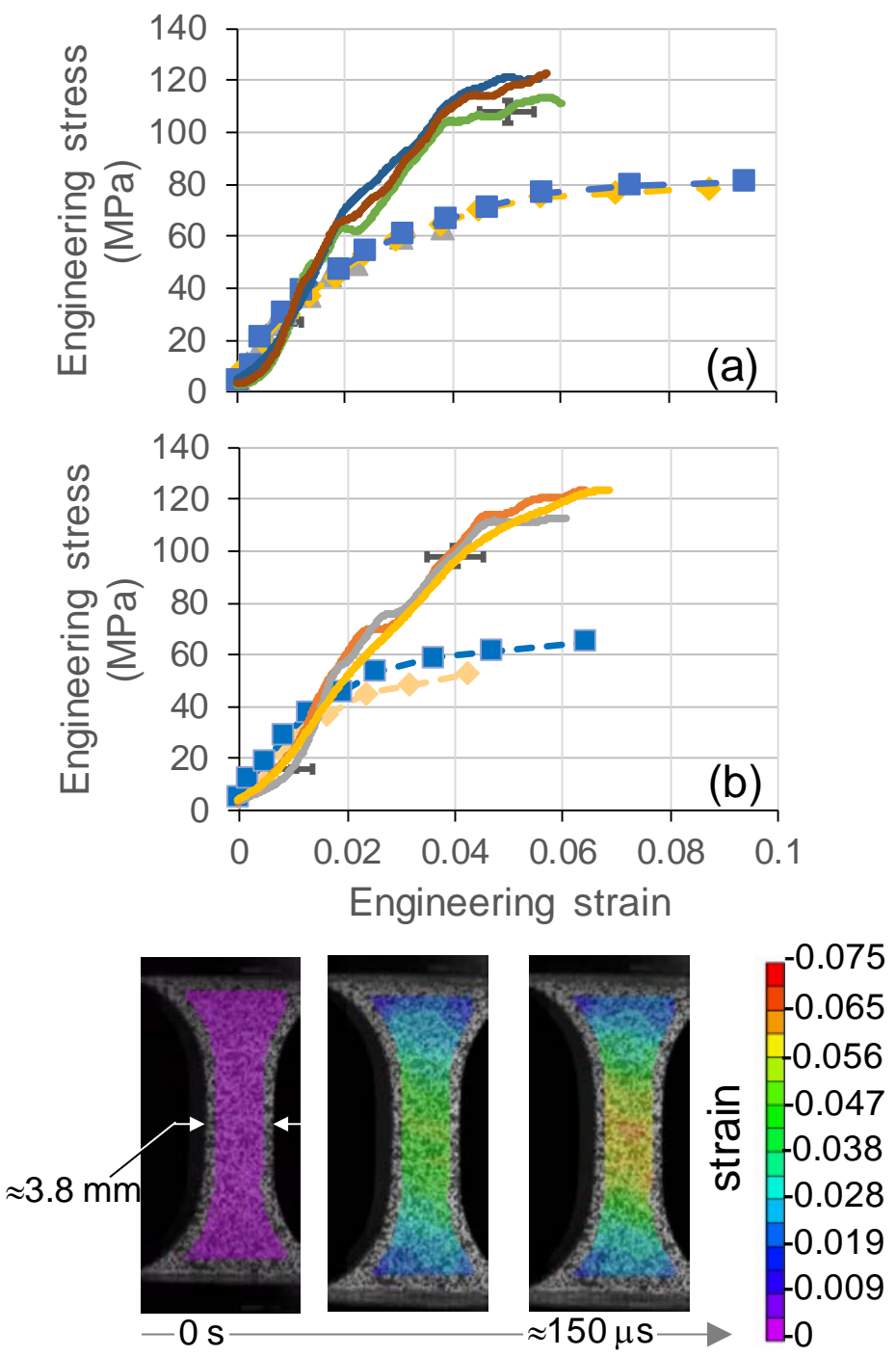

(c)

Figure 2. Typical stress-strain behaviors of the single fiber composites for the $17 \%$ DGEBD - epoxy (a) and 34 \% DGEBD - epoxy (b) for the quasi-static (dotted lines with symbols) and HSR (solid lines) during the fragmentation tests with 3DDIC images (c) of the HSR tests. Stress-strain curves up to reaching saturations are exhibited and individual symbols indicate replicates. Error bars on HSR tests are $\pm 4 \%$ on engineering stress and \pm 2 standard deviations of the averaged 3DDIC measured engineering strains over the gauge section. 


\subsection{Single glass fiber fragmentation behaviors}

Figure $3 a$ and $b$ show the number of fiber breaks obtained by the $17 \%$ DGEBDepoxy and $34 \%$ DGEBD-epoxy matrices at individual strain increments during the fragmentation tests. As strain rates for fragmentation tests increase, applied strains showing small numbers of fiber breaks ( $\mathrm{n} \leq 2)$ or no fiber breaks increased from $\approx 2.0 \%$ strain to $\approx$ $5.4 \%$ strain for the $17 \%$ DGEBD-epoxy matrix, and from $\approx 2.2 \%$ to $\approx 3.3 \%$ for the $34 \%$ DGEBD-epoxy matrix. If a fiber is not embedded in matrix, a fiber fails at such applied strains, therefore, the initial fiber break results of the fragmentation tests indicate loading rate sensitivity of the failure strains of the glass fibers. Apparent more fiber breaks in the HSR tests than the quasi-static tests were observed for the 34 \% DGEBD-epoxy matrix while such a trend is not clear for the $17 \%$ DGEBD-epoxy matrix. Since the applied strains for the fragmentation tests cannot reach to failure strains of epoxy matrix in order to count fiber breaks, whether saturation strains for glass fiber fragmentation increase with the higher strain rates has not been verified. Figure 3c exhibits more extensive interfacial deformation at the fiber breaks for the quasi-static test compared to the HSR test, implying the different state of interfacial shear stress. According to the laser Raman study [15], the fiber in the epoxy matrix at the early stage of the fragmentation process is elongated to the same strain for the model composite $\left(\varepsilon_{c}\right)$. Therefore, fiber tensile strength $\left(\sigma_{\mathrm{f}}\right)$ of the glass fiber for the HSR loading can be estimated to be nearly 50 to $60 \%$ higher than those for the quasi-static loading assuming a rate-insensitive fiber modulus $\left(\mathrm{E}_{\mathrm{f}}\right)$ i.e., $\sigma_{\mathrm{f}}=\mathrm{E}_{\mathrm{f}} \varepsilon_{\mathrm{c}}$.
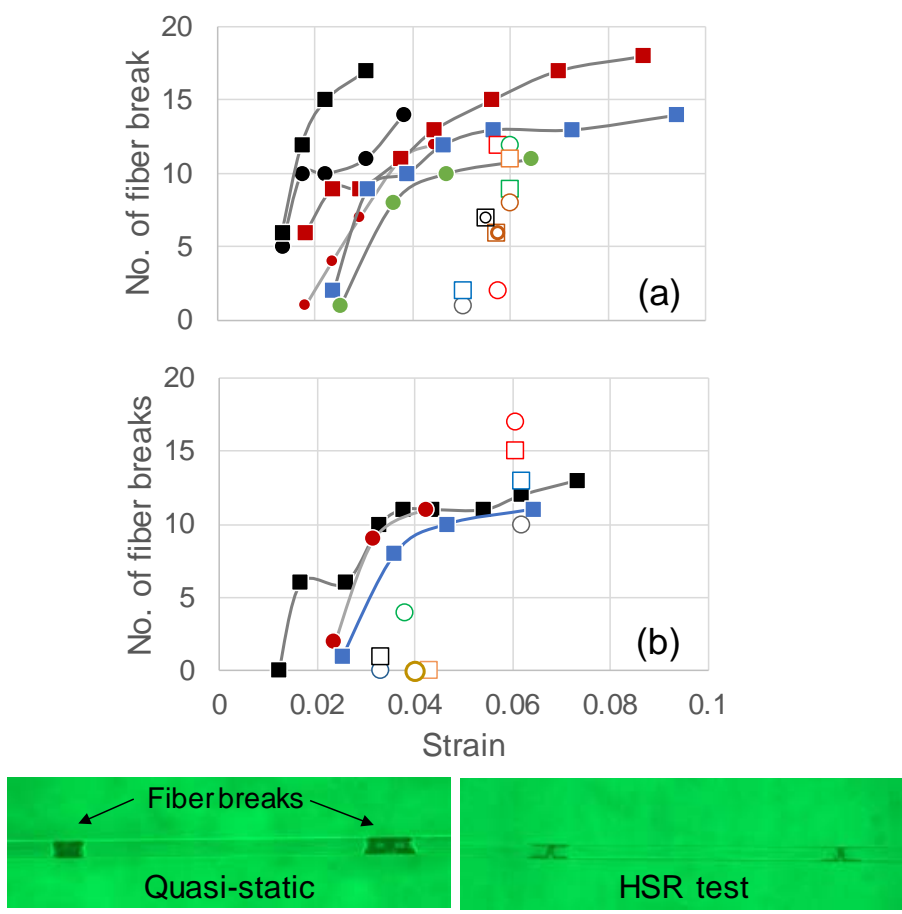

(c)

Figure 3. Evolution of the number of fiber breaks with engineering strains using the $17 \%$ DGEBD-epoxy (a) and $34 \%$ DGEBD-epoxy (b) matrix for the quasi-static (filled) and the HSR (hollow) loading conditions with the microscopic images of the fiber breaks in the $34 \%$ DGEBD-epoxy matrix (c). 
To demonstrate the effect of the strain rate on the glass fiber fragmentation behaviors, fiber break locations were statistically examined using Weibull [10] and uniform [16] distributions and the resulting goodness of fit was assessed using the probability plot correlation coefficient (PPCC) [13]. The PPCC values in Figure 4 are higher for the uniform distribution compared to the Weibull distribution indicating better fits, while the difference for the PPCCs between the quasi-static and HSR tests are almost indistinguishable. Although a smaller number of break locations were analyzed here than in Ref. [16], the consistency of the superior fit with a uniform distribution for break locations suggests an empirical stochastic rationale for the use of the specimen geometry selected for this study.

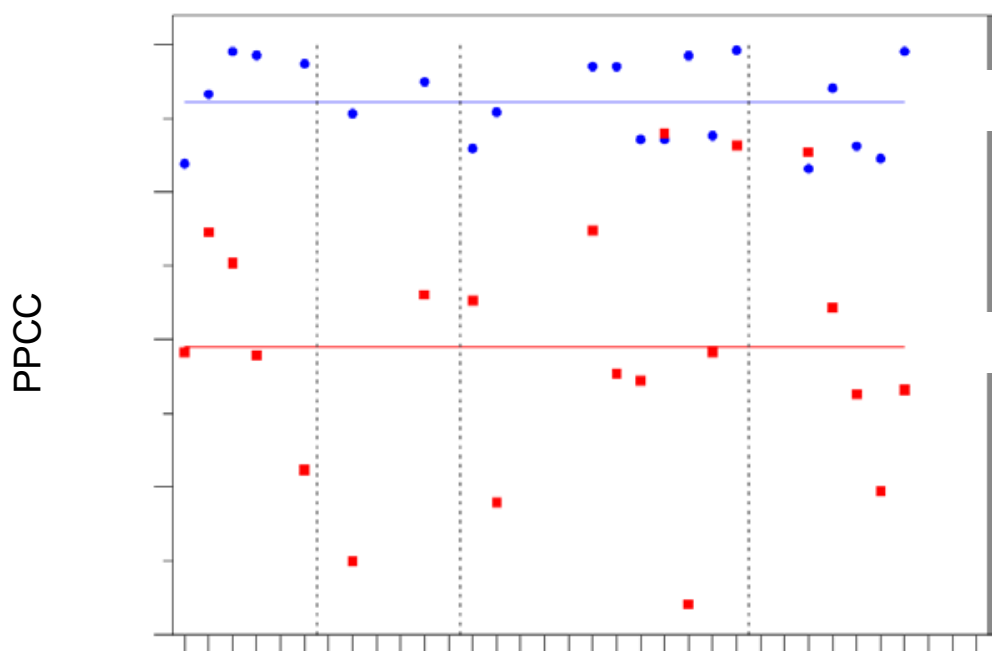

(a)

Figure 4. Probability Plot Correlation Coefficient (PPCC) comparison for the uniform (blue) and 3-parameter Weibull (red) distributions of the $17 \%$ DGEBD-epoxy (a, c) and $34 \%$ DGEBD-epoxy (b, d) specimens under the quasi-static (a, b) and HSR (c, d) are shown with the lines for the average PPCC obtained by the entire tests. Fragmentation tests with fewer than five breaks are omitted for the PPCC comparison due to insufficient sample size.

\section{Conclusions and Future Work}

The tensile behavior for the modified epoxy matrices (17 \% and $34 \%$ ) exhibited nearly $50 \%$ higher stress levels at the HSR loadings compared to the quasi-static loadings. In these epoxy matrices, the initial fiber breaks occurred around $2 \%$ strain under the quasistatic loading, while the similar number of the fiber breaks were observed between $4 \%$ to $5 \%$ strain regime under the HSR loadings. This suggests that the failure strains of the glass fibers increase with the increased strain rates although epoxy matrix significantly stiffens, which demonstrate rate dependent properties for both glass fiber and epoxy. Although the uniform distribution (PPCC of 0.99) fitted better than the 3-parameter Weibull for the fiber break locations, the fiber break locations between the two loading conditions were apparently indistinguishable in statistical comparison, which could impact on modelling for the unidirectional glass fiber/epoxy composite systems. Future work is needed to measure the glass fiber fragmentation behaviors in the epoxy matrices while the specimens are elongated 
at the HSR loadings, which will improve determing the initial and saturation stages of the glass fiber fragmentation processes.

\section{Acknowledgements}

The authors thank Stefan Leigh at NIST for his comments in preparation of this manuscript. 


\section{References}

[1] Kim YJ (2017) Use of Fiber-Reinforced Polymers in Highway Infrastructure. NCHRP Topic 47-12. https://doi.org/10.17226/24888

[2] Stewart R (2010) Automotive composites offer lighter solutions. Reinforced Plastics 54(2):22-28.

[3] Jacob GC, Starbuck JM, Fellers JF, Simunovic S, Boeman RG (2004) Strain rate effects on the mechanical properties of polymer composite materials. Journal of Applied Polymer Science 94(1):296-301.

[4] Shokrieh MM , Omidi MJ (2009) Tension behavior of unidirectional glass/epoxy composites under different strain rates. Composite Structures 88(4):595-601.

[5] Lifshitz JM (1976) Impact strength of angle ply fiber reinforced materials. Journal of Composite Materials 10(1):92-101.

[6] Naik NK, Yernamma P, Thoram NM, Gadipatri R, Kavala VR (2010) High strain rate tensile behavior of woven fabric E-glass/epoxy composite. Polymer Testing 29(1):1422.

[7] Lidgett M, Brooks R, Warrior N, Brown KA (2011) Virtual modelling of microscopic damage in polymer composite materials at high rates of strain. Plastics Rubber and Composites 40(6-7):324-332. https://doi.org/10.1179/1743289810y.0000000007

[8] ASTM International (2017) Standard Test Method for Tensile Properties of Polymer Matrix Composite Materials.D3039/D3039M-17, West Conshohocken, PA, https://doi.org/https://doi.org/10.1520/D3039_D3039M-17

[9] Herrerafranco PJ , Drzal LT (1992) Comparison of methods for the measurement of fiber matrix adhesion in composites. Composites 23(1):2-27. https://doi.org/10.1016/0010-4361(92)90282-y

[10] Shioya M , Takaku A (1995) Estimation of fiber and interfacial shear-strength by using a single fiber composite. Composites Science and Technology 55(1):33-39. https://doi.org/10.1016/0266-3538(95)00078-x

[11] McCarthy ED, Kim JH, Heckert NA, Leigh SD, Gilman JW, Holmes GA (2015) The fiber break evolution process in a 2-D epoxy/glass multi-fiber array. Composites Science and Technology 121:73-81.

[12] GRAY III GT (2000) Classic split Hopkinson pressure bar testing. ASM handbook 8:462-476.

[13] NIST (Engineering Statistics Handbook. Available at http://www.itl.nist.gov/div898/handbook/eda/section3/ppccplot.htm.

[14] Liu XM, Thomason JL, Jones FR (2008) XPS and AFM study of interaction of organosilane and sizing with e-glass fibre surface. Journal of Adhesion 84(4):322338. https://doi.org/10.1080/00218460802004386

[15] Melanitis N, Galiotis C, Tetlow PL, Davies CKL (1993) Monitoring the micromechanics of reinforcement in carbonfiber / epoxy resin systems. Journal of Material Science 28(6):1648-1654. https://doi.org/10.1007/bf00363362

[16] Kim J, Leigh S, Holmes G (2009) E-Glass/DGEBA/m-PDA Single Fiber Composites: New Insights into the Statistics of F Fiber Fragmentation. Journal of Polymer Science: Part B: Polymer Physics 47(23):2301-2312. https://doi.org/10.1002/polb.21818 


\section{Appendix A: Supplemental Materials}

Typical force and strain histories as function of times for the fragmentation tests under the quasi-static and high strain rate loading conditions 


\section{(Supplement) Fragmentation tests under the quasi-static and high strain rate loading conditions}

For the HSR loading experiments using a direct tension Kolsky bar, dual momentum traps were employed to apply a single high rate loading pulse by removing the elastic waves transmitted through and reflected from the specimen after the initial impact. In addition, the momentum traps in the HSR test eliminate elastic wave reverberations in the Kolsky bar and thereby prevent additional, uncontrolled loading on the specimen after the initial $6 \%$ strain experiment is completed. Three dimensional high speed digital image correlation (3DDIC) was used to measure the strain distribution in the gage section during dynamic loading. The 3DDIC parameters are as follows: 90000 frames/s, $1 \mu$ s exposure, (128 by 288) pixels, 15 pixel correlation subset with a 3 pixel offset, 5.66 line pairs per mm optical resolving power, and $0.07 \mathrm{~mm}$ per pixel. Strains reported are averaged over the gauge section of the specimen, and the uncertainty reported represents one standard deviation of the strain over the gauge section. A piezo electric dynamic load cell was used to provide higher resolution measurements of specimen force than were available from the transmitter bar strain gage data. The load cell was calibrated under static load and showed a maximum deviation of $50 \mathrm{~N}$ at a load of $1000 \mathrm{~N}$, or $5 \%$. The dynamic force measurements from the load cell also showed good agreement with the albeit noisy transmission strain gage data.

Figure 1S (a, b) demonstrate typical force-strain curves as a function of time for the fragmentation tests under the quasi-static and HSR loading conditions. For the quasi-static loading condition, the fragmentation test was stopped when a fiber break did not occur with increasing as the strain as shown in Figure 1S (a). For the HSR loading condition, the monotonic increment of the loading was applied to the pre-set strain. After applying the HSR loadings, the number of the fiber break was observed under the microscope with $20 \mathrm{x}$ objective lens independently.
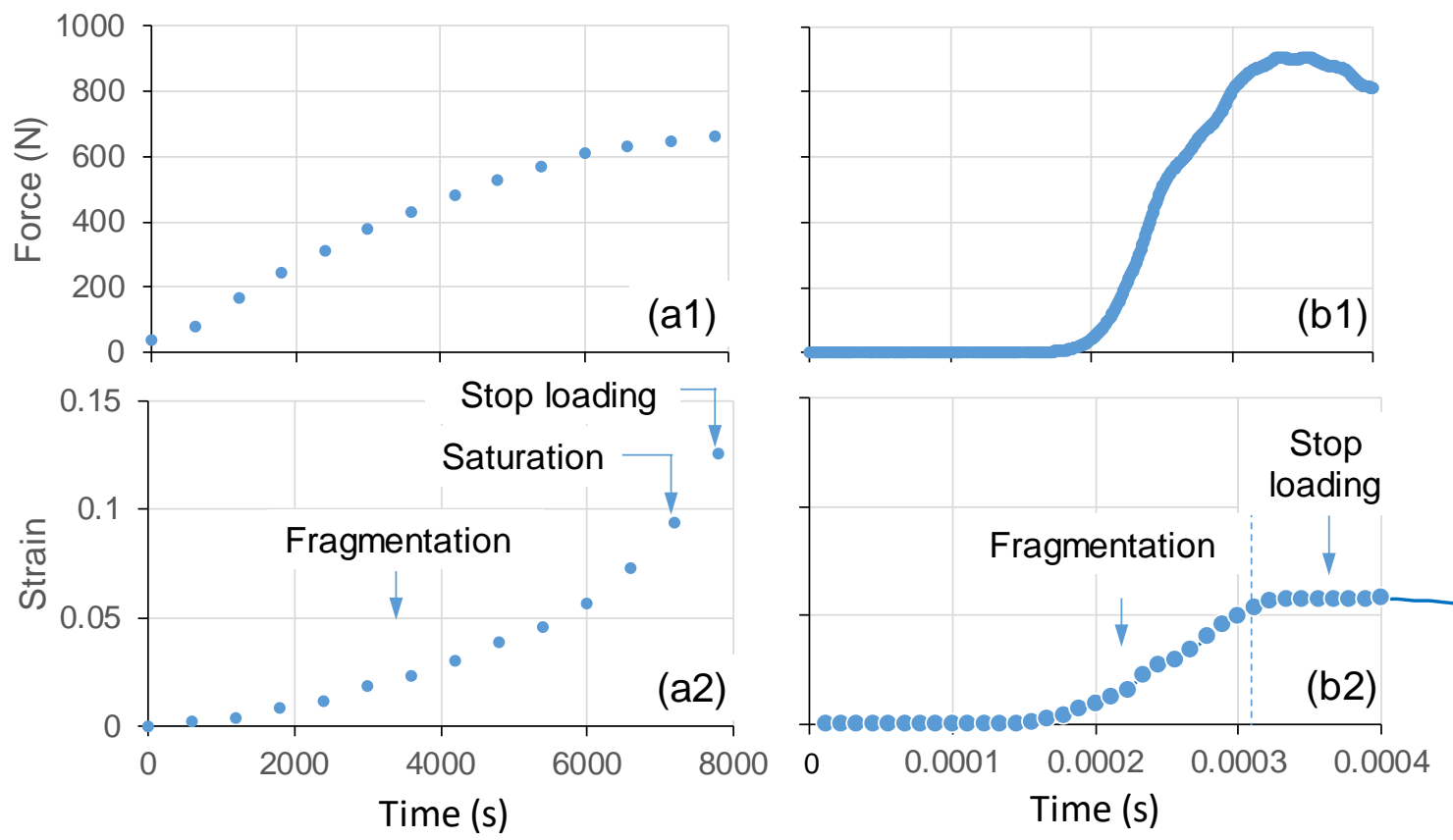

Figure 1S. Typical force-strain histories for the fragmentation tests under the quasi-static (a1 and a2) and HSR loading (b1 and b2) conditions are demonstrated as function of times. 